\title{
Introduction to the Special Issue on Clinical Psychology and Behavioral Science: Processes, Principles, and Analytic Strategies
}

\author{
Yvonne Barnes-Holmes $^{1} \cdot$ Dermot Barnes-Holmes ${ }^{2} \cdot$ Ciara McEnteggart $^{1} \cdot$ Michael J. Dougher $^{3} \cdot$ Carmen Luciano $^{4}$
}

Published online: 17 November 2020

(C) Association for Behavior Analysis International 2020

This special issue has as its focus the relationship between behavioral science and clinical psychology. Clinical psychology encompasses many schools of thought, one of which is behavioral science. This wing encompasses a broad range of interests, including the use of applied behavior analysis (ABA) such as in developmental disabilities and clinical behavior analysis (CBA) in psychotherapy. The eight conceptual articles in the current issue focus on one or both of these areas. One of the key themes of these articles is an attempt to articulate the links among the authors' clinical interests and existing or new concepts and practices in behavioral science.

Three articles contribute to issues that are directly relevant to ABA. First, the article by Greer attempts to advance behavior analysis in general by offering the concept of learned reinforcement as the selector of behavior. In particular, Greer describes how the learning of new reinforcers results in the development of verbal operants that may significantly influence the practice of ABA. Second, the article by Dixon et al. offers new tools to ABA therapists by exploring the benefits of acceptance and commitment training or therapy (ACT) for ABA. Third, the article by Kavanagh, Barnes-Holmes, and Barnes-Holmes focuses on the behavioral processes involved in relational perspective-taking and false belief. In doing so, the article offers an alternative approach to traditional mainstream views of this subject, which is likely to be of benefit to ABA practitioners.

The five remaining articles contribute to CBA. First, the article by Barnes-Holmes, Barnes-Holmes, and McEnteggart presents an updated version of Relational Frame Theory

Yvonne Barnes-Holmes

yvonne@perspectivesireland.ie

1 Perspectives Ireland Consulting Psychologists Ltd., County Louth, Ireland

2 Ulster University, Ulster, Northern Ireland

3 University of New Mexico, Albuquerque, NM, USA

4 University of Almeria, Almeria, Spain
(RFT) and its implications for process-based psychotherapy. Second, Callaghan and Follette present Interpersonal Behavior Therapy (IBT), highlighting the importance of functional-analytic assessment and adherence to behavioral principles in clinical interventions. Third, Ong, Twohig, and Levin's article presents process-based cognitive behavioral therapy (PB-CBT) as a transtheoretical approach for improving mental health service provision. Fourth, Zettle highlights the importance of targeting relevant processes and using both process-based and outcome measures in the context of singlesubject designs and evidence-based practice. These issues are directly relevant to the contemporary debate concerning the use of treatment manuals, and the distinctions and relative merits of a priori versus post-hoc manuals. Fifth, the article by Vlaeyen et al. advocates for single-subject designs and their integration with scientist-practitioner interests.

In reflecting upon all eight articles, it may be useful to return to the title of the special issue and in particular to the use of two terms: "process" and "principle." These terms often appear to be used interchangeably in the literature. Given the extent to which these terms feature in contemporary conversations in clinical psychology and in the search for new clinical treatments (see Hofmann \& Hayes, 2019), perhaps the special issue should begin by considering whether a distinction between the two terms should be made. That is, what is the difference, if any, between a process and a principle?

In psychology, the term "process" is used in many different ways, including as a mental process, a brain process, a behavioral process, and a social process. In contrast, the term "principle" has been more strongly and traditionally associated with the field of behavior analysis (as in "principles of behavior analysis"; e.g., Grant \& Evans, 1984). Why has behavior analysis so often employed the term "principle" rather than "process?" We contend that processes do not require analyses, but principles do. Insofar as we are correct, the distinction between processes and principles seems to be fundamentally important to the contemporary conversation about the importance of basic processes in the assessment and treatment of human psychological suffering. 
The term "process" seems to be used to refer to psychological change that occurs independently from any form of analysis. For example, "awareness" may be referred to as a process that occurs whether or not it is being analyzed for any given purpose. In contrast, a principle seems to require some specification of the act of the analysis by the scientist or clinician. It is interesting that Catania (1979) hinted at this distinction within behavior analysis between "operation" and "process." For example, he argued that reinforcement as an operation involves specifying a contingency between responding and consequences; reinforcement as a process involves a change in the response pattern that has been shown to be due to that contingency and not to some other factor. Combining operation and process renders the term "principle" more appropriate, at least within behavior analysis. In particular, claiming that behavior changed because of a contingency requires some form of analysis to determine if that was in fact the case. In other words, a principle requires that you demonstrate prediction and influence over a process. It is critical, therefore, that a principle requires that you specify a variable or variables that allow you to control a process; the term "process" alone does not require such specification.

Going forward, it may be important to more clearly recognize this distinction between process and principle in light of the call for process-based therapy. A process-oriented field of clinical psychology may be an improvement upon one based on DSM categories, but if it remains largely process-based, without a focus on principles, a significant risk seems to emerge. In particular, there may be a tendency to focus on proving which process-based model is the best at the expense of identifying variables that allow you to control or change those processes. In simple terms, process-based therapy is not the endgame. Principle-based therapy would be the endgame because the scientific units of analysis would specify what specific conditions need to be targeted or manipulated to produce the desired behavioral change.

Authors' Note This manuscript was prepared by Yvonne Barnes-Holmes, Dermot Barnes-Holmes, and Ciara McEnteggart with the support of the FWO Type I Odysseus Programme at Ghent University, Belgium.

\section{Compliance with Ethical Standards}

Conflict of Interest None of the authors have a conflict of interest regarding any aspect of this manuscript.

\section{References}

Catania, A. C. (1979). Learning. Englewood Cliffs, NJ: Prentice Hall. Grant, L., \& Evans, A. (1984). Principles of behavior analysis. Englewood Cliffs, NJ: Prentice Hall.

Hofmann, S. G., \& Hayes, S. C. (2019). The future of intervention science: Process-based therapy. Clinical Psychological Science, 7(1), $37-50$.

Publisher's Note Springer Nature remains neutral with regard to jurisdictional claims in published maps and institutional affiliations. 